\title{
Neurobehavioral Effects of Prenatal Alcohol: Part II. Partial Least Squares Analysis ${ }^{1}$
}

\author{
PAUL D. SAMPSON,* ANN P. STREISSGUTH, $\dagger \ddagger \S^{2}$ HELEN M. BARR $\dagger$ \\ AND FRED L. BOOKSTEINף
}

*Department of Statistics, †Department of Psychiatry and Behavioral Sciences $\Varangle$ Alcoholism and Drug Abuse Institute

$\$ C h i l d$ Development/Mental Retardation Center of the University of Washington, Seattle, WA 98195 and the ${ }^{\top}$ Center for Human Growth and Development of the University of Michigan, Ann Arbor, MI 48109

\author{
Received 16 November 1987
}

\begin{abstract}
SAMPSON, P. D., A. P. STREISSGUTH, H. M. BARR AND F. L. BOOKSTEIN. Neurobehavioral effects of prenatal alcohol: Part II. Partial Least Squares analysis. NEUROTOXICOL TERATOL 11(5) 477-491, 1989. - This paper, the second in a series of three, introduces Partial Least Squares (PLS) methods for assessing the effects of moderate levels of prenatal alcohol exposure on performance and behavior in young school-age children. Studies of human behavioral teratology pose statistical problems for which standard multiple regression methods are inadequate. Prenatal alcohol exposure, the teratogenic "dose," can be assessed only indirectly through a variety of measures of alcohol consumption. Similarly, the behavioral outcomes we examine-IQ, achievement, classroom behavior, and vigilance - are each measured indirectly in terms of multiple items or indicators. We find that a single latent variable, estimated as a linear combination of the measures of alcohol consumption, provides an appropriate measure of "dose" for summarizing the relationships between alcohol exposure and each of the four blocks of outcome variables. A pattern of alcohol consumption emphasizing binge behavior (i.e., reporting average consumption of multiple drinks per drinking occasion, or at least five drinks on any single occasion) in the period prior to recognition of pregnancy is significantly correlated with latent variables computed from each of the four outcome blocks: IQ, academic achievement, classroom behavior and attention/vigilance.
\end{abstract}

$\begin{array}{llll}\text { Behavioral teratology } & \text { Alcohol teratogenesis } & \text { Fetal Alcohol Syndrome } & \text { Fetal alcohol effects } \\ \text { Longitudinal research } & \text { Pregnancy outcome } & \text { Birth defects Alcohol use } & \text { Partial Least Squares }\end{array}$

BEHAVIORAL teratology studies, like other longitudinal research projects, often present statistical problems for which standard methods are inadequate. In principle, conventional regression analysis can assess the impact of a predictor for an outcome and adjust that assessment in light of covariates. For a study like ours, with thirteen strongly intercorrelated predictors and hundreds of outcomes, no version of regression analysis, however highly modified, is satisfactory. This paper, the second in a series of three dealing with the data from the 7-year exam in our study of alcohol teratogenesis $(24,26)$, introduces latent variable procedures for analysis of the behavior and performance of these children.

In teratology research, it is important to measure both exposure and outcome accurately. Yet, in studies using human subjects, exposure is beyond the control of the investigator. In studies of smoking or use of medications, where the dose is repeated on a daily cycle, it is sufficient to measure the typical quantity used per day together with the duration of use. However, cycles of alcohol use may occur over a day, a week, or a month, or they may not exist at all. Thus, the problem of accurately assessing alcohol exposure (the independent variable) is enormous. In addition, there are problems of poor recall, denial, and the effects of pregnancy itself on drinking patterns.

When we began our longitudinal prospective studies of alcohol, we dealt with this problem by assessing alcohol consumption in a variety of ways (29). Although reliability of these scores over a one-week interval was high $(r=.89)(27)$, we soon discovered that a heavier drinker on one scale was not necessarily a heavier drinker on another scale (28). As the relative merits of one score versus another were unknown in 1974 when the study began, we

\footnotetext{
${ }^{1}$ This work was primarily supported by grant number AA01455-01-13 from the National Institute of Alcohol Abuse and Alcoholism to A. P. Streissguth and D. C. Martin, with supplemental support from the University of Washington Alcohol and Drug Abuse Institute and from the Medical Student Research Training Program of the University of Washington. The cooperation of Group Health Hospital of Puget Sound and University Hospital during the first two years of the study is gratefully acknowledged.

${ }^{2}$ Requests for reprints should be addressed to Ann P. Streissguth, Department of Psychiatry and Behavioral Sciences, University of Washington, GG-20, Seattle, WA 98195.
} 
sampled as many heavy drinkers as possible from each of the scales in selecting the follow-up cohort (29). While our early papers dealt primarily with continuous AA scores (average ounces of absolute alcohol per day), we were always aware that an AA score of 1.0 applies equally to a woman who has two one-ounce drinks of liquor every night, and to one who has 14 drinks on Saturday night. Because we anticipated that such drinking patterns might have differential effects on the fetus, we also quantified alcohol with a variety of "binge" scores which assessed additional dimensions of the temporal concentration of usage that were not reflected in the AA score (24).

These alcohol scores vary in their relationships to the different outcomes assessed at the 7-year exam. Such relationships are difficult to interpret inasmuch as the scores are not independent either statistically or functionally. $\mathrm{H}$. Wold's method of Partial Least Squares (PLS) $(33,34)$ provides a method for examining the overall impact of alcohol exposure on offspring in this complex context. Within this framework, alcohol exposure is construed as the single underlying latent variable (LV) which best explains alcohol's effects on the various outcomes explored.

In behavioral teratology, the problem of multiple scales applies to both independent and dependent variables. It is not possible to examine all behaviors in offspring, and no body of literature suggests which are the "best" behaviors to study. The studies typically involve a variety of outcomes. Although we give these outcomes different names, their substantial correlations suggest that some underlying (latent) dimensions are being measured by them all. The methods developed for the present paper express the common dependence of the outcome variables upon the alcohol predictors by reference to latent variables underlying both sets of measures.

Part I of this trilogy (24) reviews the literature on alcohol teratogenesis and describes the outcome variables of the present study and the rationale for their selection. In the current paper, we demonstrate how the PLS method permits a single analysis of 13 alcohol predictor variables and 43 outcome variables deriving from four types of behavioral assessments: standardized IQ and achievement tests, a classroom behavior rating scale, and a laboratory measure of attention. In part III of this series, we demonstrate the utility of the PLS technique for an even more complex group of 158 neuropsychological test scores (26).

In teratology research we seek to understand the impact of a teratogen on domain specific behaviors that may be measured in different ways. Thus, although we administered an IQ test (the Wechsler Intelligence Scale for Children, Revised, WISC-R), we are more interested in performance on individual subtests than on full-scale IQ, a construct aggregating performance on many domains. PLS methods allow us to consider the relative salience of the components of IQ as they might differentially embody effects of prenatal alcohol exposure. Thus the elements of our " IQ block" are the 11 individual subtests that comprise the WISC-R. Likewise, the elements of our "Achievement block" are the three individual subtests of the Wide Range Achievement Test, Revised (WRAT-R), namely, Reading, Spelling, and Arithmetic. The Myklebust Pupil Rating Scale (PRS) provides 24 individual items for our "Classroom Behavior block," and the Continuous Performance Test (CPT) yields five scores we have previously described (25) for our "Attention block." These four outcome blocks are assessed individually, and then together in one analysis, to delineate some of the performance deficits associated with prenatal alcohol exposure. The predictor variables, 13 alcohol scores representing different aspects of prenatal alcohol exposure, constitute our "Alcohol block." These alcohol scores are combined as a single latent variable according to the size of their correlations with the outcome LV's.
Many factors other than alcohol exposure are correlated with the behaviors represented in the four outcome blocks considered here. Parental education is the factor most highly correlated with all of the outcomes. However, other exposures (for example, smoking) might conceivably produce similar performance decrements. Therefore we address also the definition and interpretation of unadjusted alcohol-outcome latent variable relationships in view of (or adjusting for) various prenatal and postnatal covariates.

\section{STLDY DESIGN AND DATA BASE}

The data analyzed here are drawn from the Seattle Longitudinal Study on Alcohol and Pregnancy. Details on the study design have been described in Part $I$ and in an earlier publication $(24,29)$. Thirteen measures of prenatal alcohol exposure were computed from maternal interviews in the fifth month of pregnancy. These measures reflect the level and pattern of drinking (including, for example, binge drinking) during two time periods: the month or so prior to pregnancy recognition (the " $P$ " variables), and midpregnancy (the " $D$ "' variables). See Part I for further details (24).

Other "independent" variables in our analyses (smoking, maternal education, etc.) may be described either as covariates or as intervening variables in the assessment of the relationship between alcohol exposure and the blocks of outcome variables. These variables have been gathered both prenatally and at subsequent exam periods

The 7-year examination included an extensive examination of 486 children in two individual testing sessions by two sets of examiners, as well as individual teacher ratings of classroom behaviors. For 454 children, there were enough valid data on each of the behavioral outcome blocks to be included in this analysis. The testing procedures are described in Part I (24). Here in Part II we analyse the standardized test results using PLS techniques; in Part III (26) we analyse a large group of neuropsychologic tests using PLS.

Missing data, always a problem in complex data sets, were handled as follows. Children entirely missing any of the four outcome blocks were deleted from the sample. For children missing only a few variables on one or more of the outcome blocks, we estimated from the rest of the block using the EM algorithm (7); these children were retained in the sample. Missing data were estimated in this way for 47 subjects.

\section{EXPLANATION AND APPLICATION OF PARTIAL LEAST SQUARES}

In this section we introduce the simplest form of Partial Least Squares (PLS) for the analysis of complex systems indirectly observed. This family of techniques is a hybrid of regression analysis and factor analysis which has recently been applied to diverse scaling problems in the natural and social sciences. For more explanations and applications of these methods, refer to $(2$, $3,15,33-35$ ).

Partial Least Squares is a method of data reduction designed for exactly the sort of data we face here: An investigator has collected two or more "blocks" of indicators and wishes to summarize the predictive interrelations among the set of all blocks considered together. Each indicator was intended to tap some aspect of a construct underlying its entire block. (For example, the construct for the alcohol items is "exposure," and for the IQ subtests "intelligence.") Yet our interest is not so much in that underlying construct (its factors, its reliability, etc.) as in its correlations with the other construct or constructs of the full data set, which are also measured indirectly via their own indicators. Regardless of the correlations among IQ subtests, different subtests are sensitive to the effects of alcohol to different extents, and also may be imagined to have been measured with more or less reliability had 
TABLE 1

CORRELATIONS BBTWEEN MEASURBS OF PRENATAL ALCOHOL EXPOSURE AND IQ SUBTEST SCORES

\begin{tabular}{|c|c|c|c|c|c|c|c|c|c|c|c|}
\hline & Inform & Similar & Arith & Vocab & Compr & DigSpan & PictComp & PictAss & BlkDes & ObjAss & Coding \\
\hline LAAP & 0.00 & -0.01 & -0.06 & 0.08 & 0.02 & -0.10 & 0.03 & -0.03 & -0.05 & 0.04 & -0.05 \\
\hline LAAD & 0.05 & 0.04 & 0.00 & 0.12 & 0.08 & -0.03 & 0.04 & 0.04 & 0.00 & 0.08 & 0.01 \\
\hline BNGEP & -0.10 & -0.06 & -0.17 & -0.02 & -0.04 & -0.14 & -0.07 & -0.08 & -0.13 & -0.02 & -0.06 \\
\hline BNGED & -0.15 & -0.11 & -0.14 & -0.07 & -0.04 & -0.13 & -0.09 & -0.01 & -0.12 & -0.01 & -0.01 \\
\hline LADOCCP & -0.04 & -0.04 & -0.07 & 0.04 & 0.03 & -0.14 & -0.05 & -0.04 & -0.07 & 0.07 & -0.06 \\
\hline LADOCCD & -0.05 & -0.03 & -0.06 & 0.04 & 0.04 & -0.13 & -0.06 & -0.03 & -0.01 & 0.11 & 0.00 \\
\hline LMAXP & -0.03 & -0.02 & -0.07 & 0.04 & 0.04 & -0.14 & -0.04 & -0.04 & -0.06 & 0.07 & -0.05 \\
\hline LMAXD & -0.05 & -0.02 & -0.07 & 0.03 & 0.02 & .0 .12 & -0.06 & -0.02 & 0.00 & 0.11 & 0.00 \\
\hline LMOCCP & 0.04 & 0.05 & 0.00 & 0.11 & 0.08 & -0.08 & 0.04 & -0.01 & -0.01 & 0.10 & -0.04 \\
\hline LMOCD & 0.09 & 0.09 & 0.03 & 0.14 & 0.12 & -0.01 & 0.04 & 0.06 & 0.05 & 0.12 & 0.01 \\
\hline QFVP & -0.03 & -0.03 & -0.07 & 0.04 & 0.04 & -0.13 & -0.02 & -0.05 & -0.05 & 0.05 & -0.06 \\
\hline QFVD & -0.01 & 0.02 & -0.05 & 0.08 & 0.08 & -0.11 & -0.03 & 0.02 & -0.01 & 0.11 & -0.00 \\
\hline ORDEXC & 0.00 & 0.00 & -0.04 & 0.06 & 0.05 & -0.12 & -0.02 & -0.04 & -0.04 & 0.10 & -0.02 \\
\hline
\end{tabular}

For other descriptive statistics on these varibles, see Part 1 of this series [24]. Note that the prefix ' $L$ ' on variable names refers to log-transformed scores.

we had the same child scored by several examiners or by the same examiner over many occasions. We therefore wish to scale the items of each block of indicators to best explain the cross-block relationships (correlations). These mutually scaled scores are the latent variables as they are constructed by PLS for two or more blocks at the same time.

Table 1 displays the ordinary correlation coefficients between each of 13 measures of alcohol exposure and each of the 11 IQ subtests from the WISC-R. This array displays a clear pattern of signs: the correlations of most of the alcohol indices with respect to most of the subtests are negative. There appears to be a stable negative correlation between alcohol exposure, as measured in this battery, and IQ as assessed by the components of the WISC-R test battery.

The simple correlations of Table 1 are measures of the degree of linear association between the alcohol scores and each of the IQ subtests. However, there is in fact no theoretical justification for linearly scaling the log-transformed AA, MAX, and ADOCC scores, the 5-point QFV scores, along with the binary BINGE variables. Using new methods of data analysis we can determine "optimal" nonlinear (monotone) transformations of the alcohol scores for prediction of the various outcomes expressed as latent variables. This methodology is explained in (21) and is based on nonlinear scaling methods now becoming increasingly common $(5,11)$. In these analyses, the optimal nonlinear scalings appropriate for prediction of each of the four outcome blocks happen to be nearly identical among themselves. We therefore use common nonlinear scalings for the alcohol scores in all our analyses. These are taken to be the optimal transformations for the relationship of the alcohol scores with the pool of 43 outcome scores from all four outcome blocks. These scalings are illustrated in Fig. 1 and the correlations of these transformed alcohol scores with the WISC-R

TABLE 2

CORRELATIONS BETWBEN TRANSFORMED MBASURES OF PRENATAL ALCOHOL EXPOSURB AND IQ SUBTEST SCORES

\begin{tabular}{|c|c|c|c|c|c|c|c|c|c|c|c|}
\hline & Inform & Similar & Arith & Vocab & Compr & DigSpm & PictComp & PictAss & BlkDes & ObjAss & Coding \\
\hline $\mathbf{A A P}$ & -0.01 & -0.03 & -0.07 & 0.05 & -0.01 & -0.10 & 0.03 & -0.04 & -0.05 & 0.02 & -0.05 \\
\hline $\mathbf{A D}$ & 0.01 & 0.03 & -0.03 & 0.09 & 0.05 & -0.05 & 0.02 & 0.03 & -0.02 & 0.07 & 0.01 \\
\hline BNGEP & -0.10 & -0.06 & -0.17 & -0.02 & -0.04 & -0.14 & -0.07 & -0.08 & -0.13 & -0.02 & -0.06 \\
\hline BNC:ED & -0.15 & -0.11 & -0.14 & -0.07 & -0.04 & -0.13 & -0.09 & -0.01 & -0.12 & -0.01 & -0.01 \\
\hline ADOCCP & -0.13 & -0.14 & -0.17 & -0.07 & -0.06 & -0.15 & -0.10 & -0.10 & -0.15 & -0.03 & -0.07 \\
\hline$A D O O C D$ & -0.14 & -0.16 & -0.11 & -0.10 & -0.03 & -0.16 & -0.10 & -0.07 & -0.11 & -0.02 & -0.02 \\
\hline MAXP & -0.12 & -0.10 & -0.17 & -0.06 & -0.04 & -0.17 & -0.10 & -0.09 & -0.11 & .0 .02 & -0.07 \\
\hline MAXD & -0.14 & -0.11 & -0.12 & -0.10 & -0.05 & -0.13 & -0.10 & -0.05 & -0.08 & 0.0 & -0.02 \\
\hline MOCCP & 0.02 & -0.03 & -0.07 & 0.06 & 0.02 & -0.10 & 0.06 & -0.06 & -0.03 & 0.08 & -0.03 \\
\hline MOCCD & 0.09 & 0.06 & 0.03 & 0.14 & 0.08 & 0.03 & 0.05 & 0.05 & 0.03 & 0.08 & -0.01 \\
\hline QFVP & -0.12 & -0.13 & -0.18 & -0.06 & -0.07 & -0.15 & -0.11 & -0.12 & -0.13 & -0.07 & -0.06 \\
\hline QFVD & -0.10 & -0.08 & -0.12 & -0.01 & 0.01 & -0.15 & -0.07 & -0.01 & -0.10 & 0.01 & 0.00 \\
\hline ORDEXC & -0.04 & -0.06 & -0.08 & 0.04 & 0.00 & -0.12 & -0.02 & -0.06 & -0.09 & 0.01 & -0.05 \\
\hline
\end{tabular}

The monotone, noaltaeer transformations of the alcohol scores were computed using methods deecribed in (21). They are illustrated in Figure 1. Becaure of these transformetions, the 'L' prefix for the log-transformed alcohol scores used in Table 1 was dropped from the variable numes used here. 

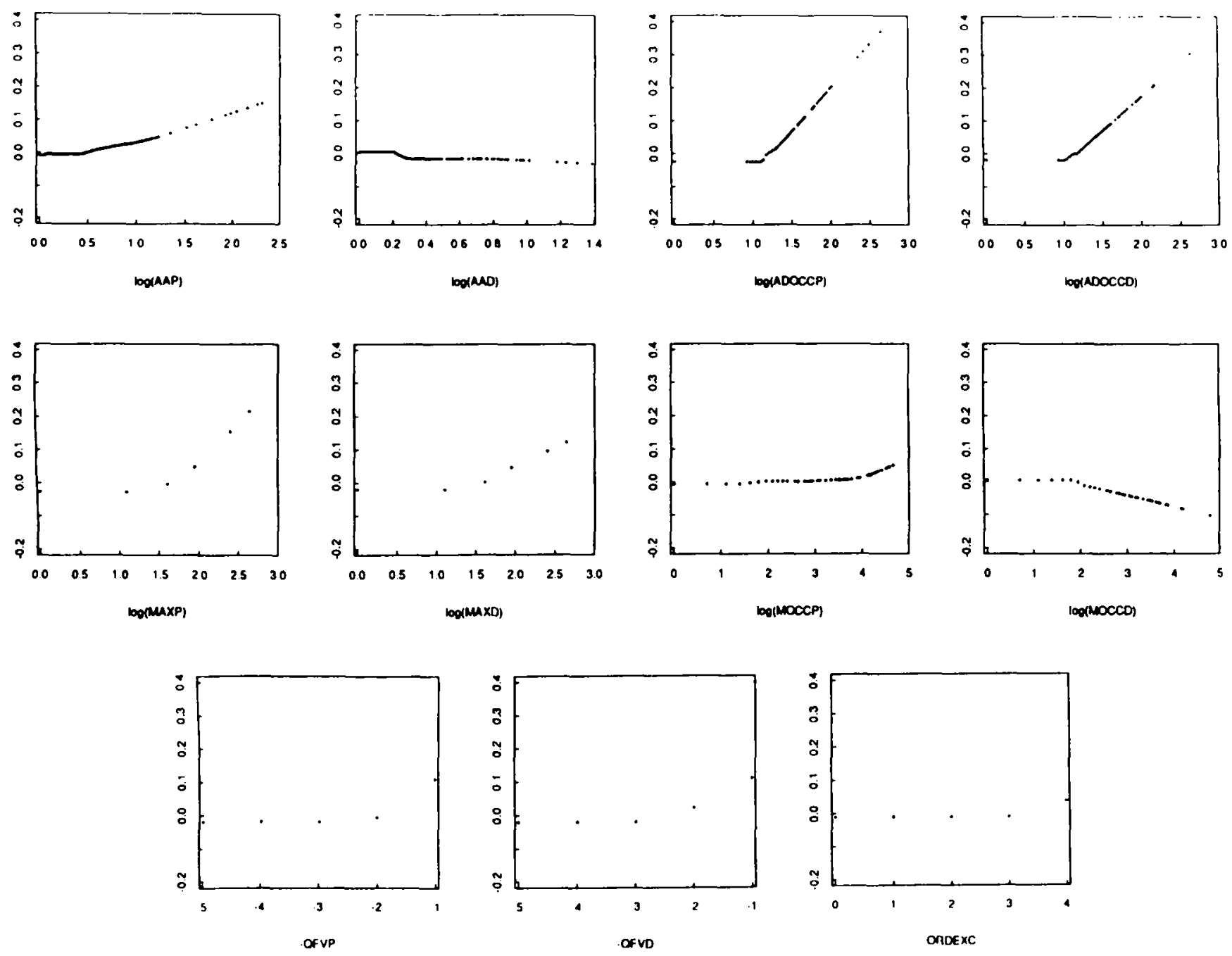

FIG. 1. Monotone nonlinear transformations of eleven of the 13 original alcohol scores (excluding the two binary BINGE measures). These were determined to optimize the correlations of the individual scores with the outcome latent variable in the large two-block analysis involving all 43 outcome scores. Details of the method are provided in (21).

subtests are given in Table 2. The pattern of correlation is now clearer and apparently stronger. All further analysis and discussion of the alcohol scores is in terms of these transformed alcohol scores.

One case corresponding to the highest AAP score $(\mathrm{AAP}=$ 25.76) originally stood out as an extreme outlier to the right on most of the initial scatterplots like those in Fig. 1. We deleted this alcohol outlier from the dataset for all computations in this part and in Par III. (Alcohol-Outcome correlations are generally lower when this case, which was diagnosed as having FAS, is excluded.)

In the Introduction we noted our original uncertainty about the appropriate mode of measuring alcohol exposure in utero, owing to lack of information at the outset of the study about the mechanism of the teratological effect. We now see that that uncertainty was justified: a variety of different measures of alcohol exposure have nearly commensurate effects on this particular block of outcome variables. Indeed, the pattern suggests that we seek an underlying construct of "net alcohol exposure," constructed as a weighted average of 13 alcohol scores, and another construct of "net intelligence deficit," constructed likewise as a weighted average, so as to interpret this pattern of correlations meaningfully. We need, therefore, a procedure for determining the weighted averages and for interpreting the extent to which their single correlation summarizes the entire pattern in Table 2.

In combining different estimates of the same quantity that vary in precision, it is standard practice to weight the contribution of each in proportion to its precision, so that the more precise estimates are given more weight in forming the average. Likewise, in attempting to construct a net score (latent variable) for alcohol exposure that is to correlate with intelligence, we should weight the alcohol measures in proportion to their correlation with the sum of the IQ subtests. This is what a Partial Least Squares procedure does.

Such a two-block analysis is typically diagrammed as shown in Fig. 2. Observed variables are indicated by squares and latent variables by circles. The single line between the two latent variables indicates our intention to explain the pattern of correlations between observables of different blocks in terms of a single pair of latent variables. We are not attempting to explain the correlations among indicators of the same block; instead we are 


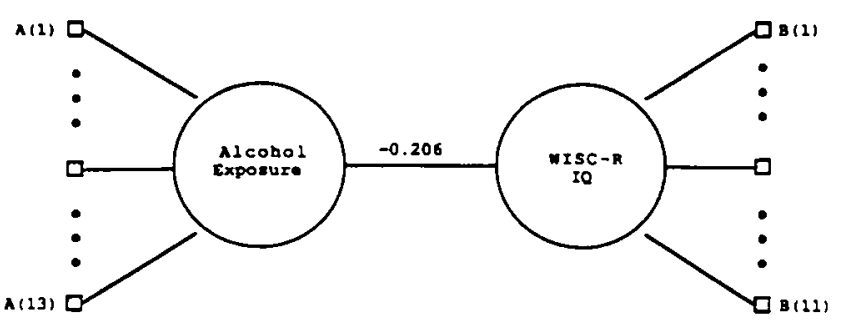

FIG. 2. Diagram for a two-block latent variable model relating 13 indicators of alcohol exposure to 11 IQ subtests from the WISC-R. The correlation between the estimated latent variables is -.206 .

determining the linear combinations of the (transformed) indicators in each block which are predictive of items in the opposite block.

The prescription just given for the latent variables of interest can be expressed simply in algebra as follows. Write the Alcohol LV score as

$$
L V_{A}=\alpha_{1} A_{1}+\ldots+\alpha_{13} A_{13}=\sum_{i=1}^{13} \alpha_{i} A_{i}
$$

where $A_{1}, \ldots, A_{13}$ are the thirteen transformed alcohol indicators, scaled to have variance one, and $\alpha_{1}, \ldots, \alpha_{13}$ are thirteen positive weights to be computed. The $\alpha$ 's are to be proportional to the correlations of the A's with a similarly defined weighted sum

$$
\mathrm{LV}_{\mathrm{B}}=\beta_{1} \mathrm{~B}_{1}+\ldots+\beta_{11} \mathrm{~B}_{11}=\sum_{j=1}^{11} \beta_{j} \mathrm{~B}_{\mathrm{j}}
$$

of the eleven IQ subtests. That is,

$$
\begin{aligned}
\alpha_{i} & \propto \operatorname{corr}\left(A_{i}, \sum \beta_{j} B_{j}\right) \\
& \propto \operatorname{cov}\left(A_{i}, \sum \beta_{j} B_{j}\right) \\
& =\sum_{j=1}^{11} r_{i j} \beta_{j}
\end{aligned}
$$

where $r_{i j}$ is the correlation of Alcohol item $i$ and IQ item $j$, the (i,j) element of the matrix $R_{A B}$ of correlations given in Table 2. Note that all of the (scaled) IQ variables are treated equally in determining the coefficient $\alpha_{i}$ of the alcohol variable $A_{i}$. The weights $\beta_{j}$ of the IQ variables are similarly required to satisfy

$$
\beta_{j} \propto \sum_{i=1}^{13} \alpha_{i} \mathbf{r}_{i j}
$$

For convenience [see equation (3) below] we scale the weights so that $\Sigma \alpha_{1}^{2}=\Sigma \beta^{2}=1$. [Note that this is not the scaling convention used by Wold (33).]

Thus each coefficient, $\alpha_{i}$ or $\beta_{j}$, is computed as a simple covariance, or regression coefficient, corresponding to an optimal least-squares prediction using part of the data. For this reason we call the linear combination $\Sigma \alpha_{i} A_{i}$ the "net partial predictor" (NPP) of $L_{B}$, denoted NPP( $\left.L V_{B} \mid A_{1}, \ldots, A_{13}\right)$. These NPP's stand in contrast to multiple regression predictors. [Wold (33) referred to these two types of predictors as "mode A" and "mode B," respectively.] Estimates of the coefficients are typically computed using an iterative algorithm, alternately updating estimates of the $\alpha_{i}$ from equation (1), and then the $\beta_{j}$ from equation (2). Such an iterative procedure constitutes a Partial Least Squares algorithm (33). The algorithm may be summarized conveniently as follows:

0. Initialize $L V_{A} \propto\left(A_{1}+A_{2} \ldots+A_{13}\right)$; that is, set $\alpha_{1}=\ldots$. $\alpha_{13}=1 \sqrt{1 \hat{3}}$.

1. Compute the linear combination of 11 IQ subtests as $L V_{B}=\Sigma \beta_{j} B_{j}=\operatorname{NPP}\left(L V_{A} \mid B_{1}, B_{2}, \ldots, B_{11}\right)$, where each $\beta_{j}$ is defined as in equation (2) with $\Sigma \beta_{j}^{2}=1$.

2. Compute the linear combination of 13 alcohol scores as $L V_{A}=\Sigma \alpha_{i} A_{i}=\operatorname{NPP}\left(L V_{B} \mid A_{1}, A_{2}, \ldots, A_{13}\right)$, where each $\alpha_{i}$ is defined as in equation (1) with $\Sigma \alpha^{2}=1$.

3. Return to 1. and iterate until $\mathrm{LV}_{\mathrm{A}}$ and $L V_{B}$ fail to change to some preset tolerance.

A computer program which implements this algorithm is available (16).

In the Appendix we describe an altemate, noniterative numerical approach to solving the equations (1) and (2) expressed in matrix notation. There are, in fact, eleven mutually uncorrelated sets of coefficients $\alpha_{i}$ and $\beta_{j}$ which satisfy these equations. (The number 11 is the minimum of the number of alcohol variables and the number of IQ variables.) The particular linear combinations $L V_{A}$ and $L V_{B}$ we want are those which have the greatest covariance. When the coefficients are scaled so that $\Sigma \alpha_{i}^{2}=\Sigma \beta_{j}^{2}$ $=1$, one can show that the sum of the squared covariances for the eleven pairs of solutions is equal to the sum of the squared correlations in the matrix $R_{A B}$. We thus refer to the ratio

$$
\frac{\left[\operatorname{cov}\left(\sum_{i=1}^{13} \alpha_{i} A_{i}, \sum_{j=1}^{11} \beta_{j} B_{j}\right)\right]^{2}}{\sum_{i=1}^{13} \sum_{j=1}^{11} r_{i j}^{2}}
$$

as the "proportion of the sum of squared cross-block correlations explained by the latent variables $L V_{A}$ and $L V_{B}$." For these data, the correlation between the latent variable scores $\Sigma \alpha_{i} A_{i}$ and $\Sigma \beta_{j} B_{j}$ is -.206 using the weights listed in Table $3 a$ (note that this correlation exceeds the largest correlation in the original correlation matrix), and $86 \%$ of the total squared cross-block correlation is explained by this first pair. The remaining information refers to correlations between all patterns of alcohol scores and IQ subtests that vary in sign. It is important to note that the latent variables were computed to maximize their covariance, not their correlation.

Those readers familiar with the multivariate method of canonical correlation analysis (18) should note how it differs from the PLS procedure presented here. Canonical correlation analysis yields "canonical variables" (not "latent variables") with greater correlation than that between $L_{\mathbf{A}}$ and $L_{\mathbf{B}}$ expressed above. Canonical correlation may be explained in terms of multiple regression: canonical variable coefficients $\alpha_{i}$ and $\beta_{j}$ would be computed as multiple regression coefficients rather than the simple regression coefficients of the NPP's in equations (1) and (2) for this PLS solution. However, the idea of multiple regression is inappropriate here. We should not compute the regression of any IQ item, or any weighted combination of items, on the alcohol variables taken as thirteen separate predictors. This is because we cannot imagine the partial effect on an IQ subtest of changing one alcohol variable while holding constant the values of all other alcohol scores. The alcohol scores covary jointly as different 
aspects of a single underlying exposure scale (an underlying factor), and so we cannot hold other alcohol scores unchanged when we vary one of them. (The Appendix provides the common noniterative characterization of canonical correlation using matrix notation along with the corresponding characterization for the NPP analysis.)

Individual coefficients reflect the relative salience of different alcohol scores in computing a latent variable score, but they are not computed as partial regression coefficients. The situation is unlike that in multiple regression where adding or deleting predictor variables can drastically alter coefficients of other predictors. In PLS analyses using the NPP, coefficients are far more robust against changes in the list of predictors (2), and also against resampling of cases in the data set.

To complete this discussion we provide in Table 4 the results of a canonical correlation analysis. In stark contrast with the PLS two-block analysis, which yielded one dominant pair of latent variables, there are three pairs of canonical variables with very similar canonical correlations. In such a case the canonical variable coefficients are not estimated at all precisely. If we tried to interpret these coefficients we would be faced with a variety of contrasts which are probably meaningless artifacts of multicollinearity in the alcohol block in view of the simple correlation structure in the matrix $R_{A B}$

The appropriate analogy for PLS is instead with the multivariate method of principal components analysis. Principal component analyses are judged by the "proportion of (total) variance explained" by successively extracted components. In the case of our PLS latent variable analysis, successive pairs of solutions to equations (1) and (2) determine scores that represent mutually independent (that is, uncorrelated) aspects of interblock prediction. Successive pairs of scores (linear combinations of scaled indicators) have successively smaller covariances. We interpret the total of the squared correlations in $\mathrm{R}_{\mathrm{AB}}$ as the total predictability of either block for the other, and each successive pair of latent variables may be said to account for an appropriate fraction of this total, as expressed in equation (3).

Note, however, that the PLS factors computed do not necessarily bear any relation to principal components of the blocks considered separately. For instance, the first principal component of the IQ block weights the subscales of Verbal IQ nearly equally, but the PLS analysis selects primarily Digit Span and Arithmetic as expressing the dependence on alcohol. PLS latent variables "explain" the cross-correlation matrix $\mathbf{R}_{\mathrm{AB}}$ in the sense just described; they are not computed to explain the within-block correlation matrices $R_{A A}$ and $R_{B B}$ as principal components do. This focus on cross-block correlation or prediction, together with the lack of assumptions on the structure of within-block correlations, distinguishes PLS analysis from the relatively better known "linear structural relations" modeling approach provided by the LISREL computer package (4); this comparison is dealt with at length in (3).

Finally, the correlation we have arrived at, -.206 , should not be corrected for "attenuation" at any stage, in spite of the custom of correcting so in the course of estimating two-block models by maximum-likelihood methods as provided in LISREL (2). Correction for attenuation is based on an assumption that the indicators of each block are modeled by the underlying factor only, together with error. We have imposed no such model, nor any other assumptions about the within-block factor structure; hence there is no way to compute an appropriate amount of attenuation. There is no reason to believe that any other estimate of the underlying scale of alcohol exposure would correlate any better than this -.206 with combinations of the IQ subtests or with any other estimate of IQ.
For these data, as shown in Table 3a. the Alcohol LV is weighted most heavily on the binge-oriented measures BINGE. ADOCC, MAX, and QFV, with greater emphasis on the prepregnancy recognition period. The AA and MOCC variables, measures of average consumption level, are clearly not consistent with the general pattern of negative correlations for the items reflecting the dimension of alcohol exposure of interest. Also, the IQ items vary in the extent to which they are predicted by alcohol as evidenced by a range of (scaled) $\beta$ 's between .07 (Object Assembly) and .47 (Digit Span).

\section{Statistical Inference: Assessing Significance}

We may assess the precision or significance of our estimated latent variable relationships using nonparametric "bootstrap" calculations $(7,9)$. Bootstrap standard errors reflect the sampling variability in the data for the given set of indicators; they do not incorporate effects of altering the lists of indicators of the underlying constructs. They are computed by repeatedly generating "bootstrap data sets" as samples of $n$ subjects drawn with replacement from the original data set of size $n$. Latent variable coefficients are computed for each of a large number (generally at least 100) of bootstrapped data sets and the latent variable relationship is judged "significant" if the latent variable coefficients are well defined (just as the test of significance of a multiple correlation coefficient is equivalent to a test of significance of the regression coefficients). The latent variable coefficients in Tables $3 a$ and $b$ are accompanied by bootstrap standard errors based on 100 bootstrap replications. These show that the estimated coefficient vector for IQ is well defined with a number of coefficients positive and relatively precisely estimated (Digit Span, Arithmetic, Information, and Similarities from the Verbal Scale; Block Design from the Performance Scale). The same is true for the Alcohol LV coefficient vector.

A cautionary remark is in order concerning statistical tests of significance. The correlation estimates should not be manipulated naively together with bootstrap standard errors (for example, by considering an estimate plus or minus twice its standard error) to test a null hypothesis of zero correlation. The PLS correlation that we compute is most biased in the case where the "true" off-diagonal correlation matrix $R_{A B}$ is identically zero; the expected value of the sample correlation of the computed LVs is always greater than zero. (The same is true for canonical correlations.) Furthermore-and in contrast to canonical correlation analysis - the latent variable correlation is not the quantity being optimized in our analysis. It is but an ancillary descriptive statistic for our explanation of structure in a cross-correlation matrix in terms of latent variables. Thus, rather than test a correlation against zero, we wish to determine whether or not the coefficient vectors defining the latent variables are well defined. A formal test of significance can be based on bootstrap methods and the "singular value decomposition" described in Appendix A, but it is beyond the scope of this paper. For present purposes, it is sufficient to notify the reader whenever the significance of an estimated Alcohol-Outcome relationship is uncertain.

\section{Covariate Adjustment}

Having established that a latent variable in alcohol exposure is related to a latent variable underlying behavioral variables measured at the age of 7 years, we must next ask to what extent this relationship may be spurious. If alcohol exposure were a consequence of some earlier variable(s) actually responsible for the behavioral decrements we observe, the analysis just completed would surely be misleading. It is necessary, therefore, to examine 
TABLE 3a

TWOBLOCK ANALYSES OF PRENATAL ALCOHOL EXPOSURE SCORES AND FOUR OUTOOME BLOCKS

1. IQ (WISC-R)

$\begin{array}{lcc}\text { Alocho LY Coefficients } \\ \text { AAP } & .12 & \pm .10 \\ \text { AAD } & .01 & \pm .12 \\ \text { BINGEP } & .31 & \pm .08 \\ \text { BNNGED } & .32 & \pm .08 \\ \text { ADOOCP } & .39 & \pm .07 \\ \text { ADOOCD } & .35 & \pm .09 \\ \text { MAXP } & .36 & \pm .07 \\ \text { MUXD } & .31 & \pm .08 \\ \text { MOCCP } & .07 & \pm .11 \\ \text { MOCCD } & .17 & \pm .15 \\ \text { QPVP } & .40 & \pm .08 \\ \text { QFVD } & .25 & \pm .08 \\ \text { ORDEXC } & .18 & \pm .11\end{array}$

2. Achievement (WRAT-R)

\section{YUSC-R LY Coefficients}

$\begin{array}{lll}\text { Information } \quad .37 \pm .10 & 0\end{array}$

Similarities $\quad .35 \pm .10$

Arithmetic $\quad .45 \quad \pm .09$

Vocabulary $\quad .18 \quad \pm .13$

Comprehension $.13 \pm .11$

Digil span $\quad .47 \quad \pm .08$

Pict completion $.27 \pm .11$

Pict assembly $\quad .22 \pm .09$

Block design $.36 \quad \pm .08$

Object assembly $.07 \quad \pm .10$

Coding $\quad .13 \pm .11$
Alochol LY Coefficients

AAP

AAD

BINGEP

BNGED

$A D O C O P$

$A D O O C D$

MAXP

MAXD

MOCCP

MOCOD

QRVP

QFVD

ORDEXC
$.10 \quad \pm .09$

$-.01 \quad \pm .10$

$.37 \pm .06$

$.33 \pm .06$

$.35 \pm .05$

$.33 \pm .06$

$.40 \pm .05$

$.32 \pm .05$

$.08 \pm .11$

$-.11 \pm .11$

$.33 \pm .05$

$.28 \pm .06$

$.19 \pm .07$
WRAT-R LY Coefficient:

Reading $\quad .52 \quad \pm .06$

Spelling $\quad .47 \quad \pm .07$

Arithmetic $\quad .71 \pm .07$

\begin{tabular}{|c|c|c|}
\hline LV Correlation & $\begin{array}{l}\text { simple: } \\
\text { adjusted": }\end{array}$ & $\begin{array}{l}-.206 \\
-.110\end{array}$ \\
\hline $\begin{array}{l}\text { Squared Covari } \\
\text { Totul Souned }\end{array}$ & Antion & $\begin{array}{r}.927 \\
1.074\end{array}$ \\
\hline So Explained & & $86.3 \%$ \\
\hline
\end{tabular}

"Cornvintes: examiner effect, grade of child, race of mother, prenatal maternal nutrition, matemal education, patemal education, mother figure employment, number of children oider than 5 years in the household, nursing.

\begin{tabular}{|c|c|c|}
\hline LV Correlation & $\begin{array}{l}\text { simple: } \\
\text { adjusted : }\end{array}$ & $\begin{array}{l}-.194 \\
-.117\end{array}$ \\
\hline $\begin{array}{l}\text { Squared Covari } \\
\text { Toxnlsounred }\end{array}$ & elntion & $\begin{array}{l}.541 \\
.549 \\
\end{array}$ \\
\hline \% Explained & & $98.5 \%$ \\
\hline
\end{tabular}

"Coyninteri grade of child, sex of child, prenatal matemal nutrition, matemal education, patemal education, biological mother in home, surrogate mother, number of children older than 5 years in the household. potentially confounding variables which could have produced the effect we are attributing to alcohol.

Approximately 150 variables examined in the present study could serve either as covariates that confound the relationship of alcohol to behavior or as intervening variables that mediate an established effect of alcohol upon its consequences. A recent publication of ours describes these covariates and the process used for incorporating appropriate covariates into the analysis (30). In human behavioral teratology research, certain prenatal conditions (such as maternal nutrition) and genetic determinants (such as race) are easily classified as covariates. To the extent that they are associated with both alcohol and the targeted outcomes, they may represent a serious source of bias in subsequent computations. Other covariates, such as smoking, must be examined when previous research (which may not have examined alcohol exposure) has suggested a relationship with similar outcomes (22).

Prenatal covariates of child behavior are the essential and appropriate ones to adjust for in assessing possible causal links between prenatal determinants, such as alcohol exposure, and the later behavioral outcomes. This is because it is impossible for prenatal covariates themselves to be consequences of alcohol exposure through the effects of the "outcome": the child with alcohol-induced behavioral deficits. We thus begin by adjusting our analyses for prenatal covariates (and for exam conditions when appropriate). We then consider the influence of postnatal "environmental" covariates, even though their role in the assessment of causal associations might be challenged on the grounds that postnatally determined variables should not be considered as covariates in assessing causal associations (6). We analyze covariates primarily for their effect on our interpretation of the relation- ships of alcohol to behavior; a detailed interpretation of the covariate effects on the outcomes is not an aim of this analysis. Because of intercorrelations among the covariates, effective interpretation would require their analysis in terms of latent variables also. [See (21) for an example of such a detailed analysis.] In this paper we focus on how adjustment for relevant covariates affects our interpretation of estimated Alcohol-Outcome latent variable relationships.

Broad screening of the potential covariates measured in this study led us to the following list of fundamental covariates: 1) sex, age of child (except for analysis of the WISC-R and WRAT-R which are already age adjusted), and grade of child at testing, along with examiner effects if appropriate; 2) other prenatal exposures: nicotine, caffeine, marijuana, aspirin, and antibiotics; 3) parental characteristics: paternal and maternal education, maternal age, race, prenatal nutrition and parity. Covariates measured postnatally, usually reflecting primarily the postnatal environment, included marital status (at birth and seven years), socioeconomic status, parental status (whether biological, foster, or surrogate parents are at home, whether parents are employed), breast feeding and child nutritional intake, the number of children less than and greater than 5 years of age in the household, and preschool and private school experience.

We proceed in two ways. First, following computation of latent variables using the ordinary (unadjusted) correlations, as we have just described, we adjust the latent variables (and thereby their correlations) for dependence on covariates. From the broad list of covariates provided above we selected (separately for each twoblock analysis) a subset of variables which contributed significantly to the prediction of the outcome latent variable. These were 
TABLE 3a

(CONTINUED)

3. Myklebust Pupil Rating Scale

$\begin{array}{lcc}\text { Alcohol LV Coefficients } \\ \text { AAP } & .18 & \pm .07 \\ \text { AAD } & -.04 & \pm .09 \\ \text { BINGEP } & .33 & \pm .05 \\ \text { BINGED } & .29 & \pm .05 \\ \text { ADOCCP } & .42 & \pm .04 \\ \text { ADOOCD } & .28 & \pm .06 \\ \text { MAXP } & .35 & \pm .04 \\ \text { MAXD } & .28 & \pm .05 \\ \text { MOCCP } & .12 & \pm .07 \\ \text { MOOCD } & . .16 & \pm .10 \\ \text { QFVP } & .44 & \pm .05 \\ \text { QFVD } & .22 & \pm .06 \\ \text { ORDEXC } & .20 & \pm .07\end{array}$

\section{Myklebunt PRS LV}

Compre wonds $\quad .26 \quad \pm .04$

Follows instruct $.20 \quad \pm .03$

Compre discuss $\quad .18 \quad \pm .04$

Retsins info $\quad .26 \quad t .03$

Vocabulary $\quad .19 \quad \pm .05$

$\begin{array}{lll}\text { Grammat } & .25 & \pm .04\end{array}$

Word recall $\quad .24 \quad \pm .05$

Relates exper $.21 \quad \pm .05$

Formulales ideas $.21 \quad \pm .04$

Judges time $\quad .15 \quad \pm .04$

Spatial orientation.17 \pm .04

Judges relations $\quad .15 \quad \pm .05$

Knows directions $.14 \pm .06$

Coordinalion is t.05

Balance $\quad .09 \pm .05$

Manual dexterity $.17 \pm .05$

Coop/impulsivity $.28 \quad \pm .04$

Altention $\quad .22 \pm .04$

Organization $\quad .23 \quad \pm .04$

Flexibility $\quad .20 \pm .05$

Social accepunce $.16 \pm .05$

Responsibility $\quad .20 \quad \pm .04$

Finishes tasks $\quad .22 \quad \pm .04$

$\begin{array}{lll}\text { Tactfulness } & .25 & \pm .04\end{array}$
4. Attention/Vigilence

$\begin{array}{lrr}\text { Alcohol LV Coefficient } \\ \text { AAP } & .24 & \pm .10 \\ \text { AAD } & .01 & \pm .12 \\ \text { BNNGEP } & .34 & \pm .08 \\ \text { BNGGED } & .37 & \pm .08 \\ \text { ADOCCP } & .46 & \pm .07 \\ \text { ADOOCD } & .32 & \pm .09 \\ \text { MAXP } & .37 & \pm .07 \\ \text { MAXD } & .22 & \pm .08 \\ \text { MOCCP } & .11 & \pm .11 \\ \text { MOCCD } & -.18 & \pm .15 \\ \text { QFVP } & .28 & \pm .08 \\ \text { QFVD } & .25 & \pm .08 \\ \text { ORDEXC } & .10 & \pm .11\end{array}$

Vigilance LV Coefficient:

$\log (\mathrm{XEO}) \quad .23 \quad \pm .18$

$\log (X E C) \quad .44 \quad \pm .17$

$\log$ (AXEO) $\quad .47 \quad \pm .13$

$\begin{array}{lll}\log (\mathrm{AXEC}) & .73 \quad \pm .09\end{array}$

Reaction time $-.01 \quad \pm .18$

\begin{tabular}{|c|c|c|}
\hline LV Correlation & $\begin{array}{l}\text { simple: } \\
\text { adjusted": }\end{array}$ & $\begin{array}{l}-.213 \\
-.109\end{array}$ \\
\hline $\begin{array}{l}\text { Squared Covari } \\
\text { Totnl Sounred }\end{array}$ & ation & $\begin{array}{r}3.153 \\
3.346 \\
\end{array}$ \\
\hline कo Explained & & $94.2 \%$ \\
\hline
\end{tabular}

Coyarintes: Sex of child, aspirin consumption. patemal education, mother married (at time of birth of child), number of children older than 5 year in household, child/adult mato in household.

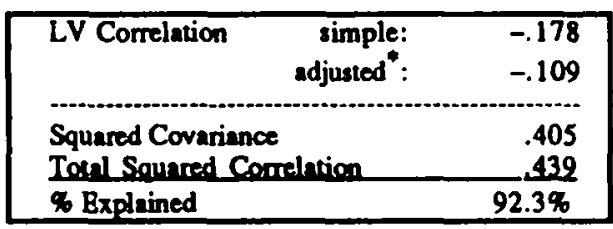

Covarintes: age of child, $\operatorname{sex}$ of child, mother's age, paternal education, socio-economic status, mother married (at time of birth of child).

${ }_{1}$ Numbers prefixed by \pm are bootstrap standard errors computed as explained in the text.

${ }^{2}$ Latent variable correlations are adjusted for the lin of covariates presented below the aummary boxes.

chosen using all-subsets-regression procedures applied first to the prenatal covariates and other exposures, then to the postnatal family environment measures. We also looked for interaction effects expressed as products of the Alcohol latent variable with these selected covariates. We thus compute the fraction of the Alcohol-Outcome correlation that can be explained in terms of the list of covariates.

In a second type of analysis, we adjust all the individual measures - both the Alcohol scores and the Outcome scores-for these selected covariates, and then compute PLS analyses for the resulting two-block partial correlation matrices just as for the unadjusted correlations before. This second analysis is more flexible in that it permits differential adjustment of the individual Alcohol and Outcome scores, which are not all equicorrelated with each of the potential covariates. For example, the patterns of alcohol consumption reflected in the set of Alcohol scores vary on average by parental education level.

\section{RESULTS OF TWO-BLOCK ALCOHOL-OUTCOME ANALYSES}

We now describe the relationships of alcohol to each of the four blocks of behavioral outcomes. In the previous section we used the IQ block to demonstrate PLS analyses; now we present the results of the PLS analyses of IQ as well as the three other outcome blocks: Achievement, Classroom Behavior and Attention. Table 3a.1-4 presents simple (unadjusted) two-block analyses together with the partial correlation between the latent variables after adjustment for the covariates listed at the bottom. Table $3 \mathrm{~b} .1-4$ presents results of two-block analyses of partial correlation matrices (for the blocks of adjusted measures) computed using the same sets of covariates listed at the bottom of Table $3 a$.

\section{Prediction of IQ by Alcohol}

The IQ block consists of the eleven scaled scores of the standard WISC-R, all age standardized in the usual fashion. The 
TABLE 3b

TWO-BLOCK PLS ANALYSES OF PRENATAL ALCOHOL EXPOSURE SCORES AND FOUR OUTCOME BLOCKS ADJUSTED FOR COVARIATES

1. IQ (WISC-R)

$\begin{array}{lcc}\text { Alohol LY Coefficient } \\ \text { AAP } & .22 & \pm .09 \\ \text { AAD } & .30 & \pm .10 \\ \text { BNNGEP } & .30 & \pm .06 \\ \text { BNGED } & .34 & \pm .13 \\ \text { ADOCCP } & .28 & \pm .07 \\ \text { ADOOCD } & .24 & \pm .09 \\ \text { MAXP } & .29 & \pm .06 \\ \text { MAXD } & .23 & \pm .09 \\ \text { MOCCP } & .26 & \pm .10 \\ \text { MOOCD } & .16 & \pm .09 \\ \text { QPVP } & .32 & \pm .07 \\ \text { QFVD } & .29 & \pm .09 \\ \text { ORDEXC } & .32 & \pm .07\end{array}$

\section{Achievement (WRAT-R)}

WISC-RLY Coefficients

Information $\quad .25 \quad \pm .17$

Similarities $\quad .25 \quad \pm .20$

Arithmetic $\quad .49 \pm .13$

Vocabulary $\quad-.12 \quad \pm .19$

Comprehension $-.05 \quad \pm .18$

Digit span $\quad .65 \quad \pm .13$

Pict completion $\quad .15 \quad \pm .19$

Pict assembly $\quad .11 \quad \pm .16$

Block design $\quad .32 \quad \pm .16$

Object susembly $\quad-.22 \quad \pm .20$

$\begin{array}{lll}\text { Coding } & .07 & \pm .19\end{array}$

\begin{tabular}{lr}
\hline LV Correlation & -.172 \\
\hline Squared Covariance & .352 \\
Toul Soluned Correlation & .451 \\
\hline Explained & $78.0 \%$ \\
\hline
\end{tabular}

*Cosprinter: examiner effect, grade of child, noce of mother, prenatal maternal nutrition, matemal education, patemal education, mother figure employment, number of children older than 5 years in the household, nursing.

$\begin{array}{lll}\text { Alcohol LV Coeffigients } \\ \text { AAP } & .12 & \pm .10 \\ \text { AAD } & .19 & \pm .09 \\ \text { BINGEP } & .42 & \pm .08 \\ \text { BNGED } & .36 & \pm .10 \\ \text { ADOCCP } & .26 & \pm .10 \\ \text { ADOOCD } & .21 & \pm .11 \\ \text { MAXP } & .39 & \pm .07 \\ \text { MUXD } & .29 & \pm .10 \\ \text { MOCCP } & .13 & \pm .12 \\ \text { MOCCD } & .15 & \pm .10 \\ \text { QFVP } & .28 & \pm .12 \\ \text { QFVD } & .30 & \pm .09 \\ \text { ORDEXC } & .30 & \pm .08\end{array}$

WRAT-RLYCoefficients Reading $\quad .48 \quad \pm .15$ Spelling $\quad .37 \quad \pm .18$ Arithmetic $\quad .80 \quad \pm .12$

\begin{tabular}{|lr|}
\hline LV Correlation" & -.122 \\
\hline Squared Covariance & .219 \\
Totsl Sonured_Comelntion & .226 \\
\% Explained & $96.9 \%$ \\
\hline
\end{tabular}

"Cevniater: srade of child, sex of child, prenatal matemal nutrition, matemal education, patemal education, biological mother in home, surrogate mother, number of children older than 5 years in the housebold. net IQ LV extracted by PLS as most appropriate for adumbrating the effects of alcohol is a combination principally weighting Digit Span and Arithmetic, with significant weights also for Information, Similarities and Block design. Four scaled scores (Vocabulary, Comprehension, Object Assembly, and Coding) show negligible association with this first latent variable. The resulting combination correlated -.206 with the weighted average of the alcohol variables shown in Table 3a.1. This pair of LV's explains $86 \%$ of the predictability between the blocks.

Father's education in years is the best predictor of the outcome latent variable in this analysis (as it is for the other three bocks as well). Adjusting the latent variables for this covariate alone results in a partial latent variable correlation of -.12 . That is, approximately two-thirds of the squared correlation between these two latent variables can be explained by their dependence on paternal education. A number of other variables, listed below Table 3a.1, also contributed significantly to the prediction of the IQ latent variable. However, collective adjustment for all of these variables reduces this correlation only slightly further, to -.11 . This remains significant according to a conventional multiple regression analysis. Further analyses of possible interaction effects suggest that the estimated alcohol effect on the IQ latent variable may be greater (and slightly more significant) in families with lower parental education and in families with a greater number of older siblings.

The two-block analysis of adjusted Alcohol and IQ scores, presented in Table $3 \mathrm{~b} .1$, focuses interpretation even more clearly on the Digit Span and Arithmetic scores as judged by the latent variable coefficients and their bootstrap standard errors. The alcohol latent variable is more evenly weighted across all the measures of consumption after adjustment. The latent variable correlation based on the adjusted variables is -.17 .

\section{Prediction of Achievement by Alcohol}

The Achievement block is represented in our data set by the three WRAT scores on Reading, Spelling, and Arithmetic, each age standardized by the usual procedure. The PLS analysis combined the Achievement scores using weights in the ratio of about 5:5:7 according to Table 3a.2. That is, the Arithmetic score is most associated with the predictive Alcohol LV. This is consistent with the analysis of the IQ block, for which the quantitative subtests were those most highly correlated with prenatal alcohol exposure. The Alcohol LV is again specified to have greatest weights corresponding to the binge-related measures BINGE, ADOCC, MAX, and QFVP. There is no question of any additional dimensionality of these latent variables: the single pair of LV's in Table 3a.2, correlated - .194, accounting for nearly $99 \%$ of all the predictability linking the blocks.

Adjustment for paternal education alone reduces the latent variable correlation to just below -.14 . Adjustment by further significant predictors, as listed below Table $3 \mathrm{a} .2$, reduces this correlation further to about -.12 (which, again, is nominally significant according to multiple regression statistics).

The two-block analysis of adjusted variables presented in Table $3 \mathrm{~b} .2$ is consistent with the adjustment of the LVs from Table 3a.2. The clearly significant latent variable coefficients are for the Arithmetic and Reading subscales. The LV correlation based on 
TABLE 3b

(CONTINUED)

3. Myklebust Pupil Rating Scale

$\begin{array}{lcc}\text { Alcohol LV Coefficients } \\ \text { AAP } & .16 & \pm .12 \\ \text { AAD } & .07 & \pm .16 \\ \text { BINGEP } & .37 & \pm .08 \\ \text { BNGED } & .32 & \pm .11 \\ \text { ADOCCP } & .39 & \pm .07 \\ \text { ADOOCD } & .19 & \pm .11 \\ \text { MAXP } & .30 & \pm .06 \\ \text { MAXD } & .28 & \pm .11 \\ \text { MOCCP } & .09 & \pm .13 \\ \text { MOCCD } & . .05 & \pm .17 \\ \text { QFVP } & .50 & \pm .09 \\ \text { OFVD } & .19 & \pm .11 \\ \text { ORDEXC } & .29 & \pm .09\end{array}$

\section{Mykleburt PRS LY}

Compre words .32

Follows instruct .12

Compre

$.15 \pm .10$

Retains info $\quad .33 \pm .11$

Vocabulary $\quad .17 \pm .10$

Grammar $\quad .28 \pm .10$

Word recall $\quad .31 \pm .12$

Relates exper $.23 \pm .10$

Formulates idens $.25 \quad \pm .08$

Judges time $\quad 07 \pm .11$

Spatial orientation.19 \pm .09

Judges relations $.08 \quad \pm .10$

Knows directions $.07 \quad \pm .12$

Coordination $\quad .16 \pm .09$

Balance $\quad .05 \quad \pm .10$

Manual dexterity $.17 \pm .10$

Coop/impulsivity $.27 \quad \pm .09$

$\begin{array}{lll}\text { Altention } & .16 \quad \pm .07\end{array}$

Organization $\quad .21 \pm .10$

Flexibility $\quad .21 \quad \pm .10$

Social acceptance $.09 \quad \pm .09$

Responsibility $\quad .18 \pm .09$

Finishes lasks $\quad .16 \quad \pm .09$

Tactfulness $\quad .26 \pm .11$
4. Attention/Vigilance

$\begin{array}{lll}\text { Alcohol LV Coefficients } \\ \text { AAP } & .24 & \pm .13 \\ \text { AAD } & .18 & \pm .11 \\ \text { BNNGEP } & .40 & \pm .07 \\ \text { BNNGED } & .40 & \pm .11 \\ \text { ADOCCP } & .40 & \pm .08 \\ \text { ADOOCD } & .31 & \pm .10 \\ \text { MAXP } & .37 & \pm .07 \\ \text { MAXD } & .18 & \pm .10 \\ \text { MOCCP } & .14 & \pm .12 \\ \text { MOCCD } & .06 & \pm .14 \\ \text { QFVP } & .18 & \pm .10 \\ \text { QHVD } & .25 & \pm .09 \\ \text { ORDEXC } & .20 & \pm .12\end{array}$

\begin{tabular}{lll} 
Vigilance LV Coefficients \\
\hline $\log$ (XEO) & -.07 & \pm .33 \\
$\log$ (XEC) & .17 & \pm .31 \\
$\log$ (AXEO) & .43 & \pm .21 \\
$\log$ (AXEC) & .82 & \pm .18 \\
Reaction time & .33 & \pm .27
\end{tabular}

\begin{tabular}{|lr|}
\hline LV Correlation & -.126 \\
\hdashline Squared Covariance & .141 \\
Totsl Squared Correlation & .174 \\
\hline$\%$ Explained & $\mathbf{8 1 . 3 \%}$ \\
\hline
\end{tabular}

"Coveriates: age of child, sex of child, mother's age, patemal education, socio-economic status, mother married (at time of birth of child).

Covarintes: Sex of child, aspirin consumption,
patemal education, mother married (at time of birth
of child), number of children older than 5 years in
household, child/adult ratio in household.

\begin{tabular}{|lr|}
\hline LV Correlation & -.118 \\
\hline Squared Covariance & .773 \\
Tonl Squared Correlation & .982 \\
\% Explained & $\mathbf{7 8 . 7 \%}$ \\
\hline
\end{tabular}

†Numbers prefixed by \pm are bootstrap standand errors computed as explained in the text.

fLatent variable correlations are computed after adjustment of the items of each block for the covariates listed below the summary boxes.

these adjusted scores is, again, -.12 .

\section{Prediction of Classroom Behaviors by Alcohol}

For the relation between the 13 alcohol indicators and the 24 Classroom Behavior scores of the Myklebust Pupil Rating Scale, the first pair of latent variables (the pair having all Classroom Behavior weights positive, and so corresponding to our general hypothesis of an "underlying" or "net" relationship) correlate -.213 and explain $94 \%$ of all the predictability there is between the blocks. The Alcohol LV is again dominated by the binge variables (with greater weight in general on the prepregnancy recognition measures); the response $L V$ assigns greatest weight to Cooperation/lmpulsivity, Comprehends Words, Retains Information, Grammar, and Tactfulness among a list of items with significant weights according to the bootstrap analysis.
Adjustment for parental education reduces the LV correlation below -.15 . Further adjustment for the covariates listed in Table 3a. 3 leads to a partial LV correlation of -.11 . For this outcome block there is some evidence that the effect of alcohol LV on the response $\mathrm{LV}$ is greater in families with mothers who were unmarried at the time of the birth of the child.

The two-block analysis of adjusted correlations between the alcohol measures and the pupil rating scale items more clearly emphasizes Comprehends Words, Retains Information, Grammar, Word Recall, Cooperation/Impulsivity, and Tactfulness. The LV correlation is -.12 .

\section{Prediction of Vigilance by Alcohol}

Once again it is essentially the same Alcohol LV which is found to channel the interblock relation: the weights for BINGE, 
TABLE 4

CANONICAL CORRELATION ANAL YSIS FOR (TRANSFORMED) PRENATAL ALCOHOL MEASURES AND IQ SUETEST SCORES

\begin{tabular}{|c|c|c|c|c|c|}
\hline \multirow[b]{2}{*}{ Coefficients } & \multicolumn{5}{|c|}{ Canonical Correlations } \\
\hline & 0.30 & 0.28 & 0.26 & 0.20 & 0.16 \\
\hline \multicolumn{6}{|l|}{ Alcohol } \\
\hline$\overline{A A P}$ & 0.37 & 0.14 & -0.35 & 0.23 & 0.08 \\
\hline AAD & -0.12 & 0.07 & -0.52 & -0.21 & -0.23 \\
\hline BINGEP & -0.08 & -0.39 & -0.41 & -0.15 & -0.14 \\
\hline BINGED & -0.13 & 0.27 & -0.09 & 0.33 & 0.01 \\
\hline$A D O C C P$ & 0.00 & -0.34 & 0.05 & 0.41 & -0.55 \\
\hline$A D O O C D$ & -0.37 & .0 .08 & 0.38 & 0.10 & 0.43 \\
\hline MAXP & -0.16 & 0.45 & 0.04 & -0.32 & 0.30 \\
\hline MAXD & 0.40 & 0.21 & -0.01 & -0.29 & -0.29 \\
\hline MOOCP & -0.56 & -0.47 & 0.26 & -0.44 & 0.05 \\
\hline MOCCD & 0.36 & -0.23 & 0.36 & 0.29 & 0.30 \\
\hline QFVP & -0.18 & 0.31 & 0.16 & -0.12 & -0.31 \\
\hline QFVD & -0.18 & -0.03 & -0.11 & -0.04 & 0.22 \\
\hline ORDEXC & 0.02 & -0.09 & 0.21 & 0.34 & 0.17 \\
\hline \multicolumn{6}{|l|}{ LO } \\
\hline Information & -0.02 & -0.48 & 0.38 & -0.20 & -0.38 \\
\hline Similarities & 0.34 & 0.20 & -0.67 & -0.50 & 0.08 \\
\hline Arithmetic & 0.41 & 0.08 & 0.25 & 0.28 & 0.48 \\
\hline Vocabulary & -0.11 & .0 .50 & -0.30 & 0.49 & -0.14 \\
\hline Comprehension & -0.36 & 0.20 & 0.33 & 0.02 & 0.41 \\
\hline Digit Span & 0.53 & 0.06 & 0.08 & 0.15 & -0.49 \\
\hline Picture Completion & -0.04 & -0.34 & -0.02 & -0.13 & 0.26 \\
\hline Picure Assembly & 0.22 & 0.31 & -0.25 & 0.42 & 0.19 \\
\hline Block Design & 0.35 & 0.21 & 0.24 & -0.40 & 0.03 \\
\hline Object Assembly & -0.33 & -0.39 & -0.09 & -0.01 & -0.02 \\
\hline Coding & -0.09 & 0.15 & -0.04 & -0.15 & 0.29 \\
\hline
\end{tabular}

ADOCC and MAX are again largest. The Vigilance scores vary in their correlations with the Alcohol LV: AXEC is most strongly correlated; the correlations of AXEO and XEC are intermediate; and reaction time and XEO are only weakly correlated with this alcohol latent variable. The correlation between the Alcohol and Vigilance LV's is -.178 , and they account for $92 \%$ of the predictability between the blocks.

In this case the partial LV correlation after adjusting for parental education is -.12 . The remaining significant correlates of the Vigilance LV listed at the bottom of Table 3a.4 reduce this correlation to -.09 . Again there is some evidence that the alcohol effect is greater in families with mothers who were unmarried at the time of the birth of the child.

A two-block analysis of adjusted alcohol measures and performance scores identifies the $\mathrm{AX}$ task, and primarily false alarms (AXEC), as most clearly reflecting prenatal alcohol exposure. This two-block LV correlation is -.13 .

\section{Relationships Among Outcomes}

We have found that essentially a single system of transforms and weights for the thirteen selected measures of alcohol exposure adequately summarizes the structure of prediction regardless of the type of outcome from among the four we consider here: IQ, Achievement, Classroom Behavior, or Vigilance at a laboratory task. The correlations of outcome LV's with this net alcohol exposure $\mathrm{LV}$ range from -.18 to -.21 ; after covariate adjustment, correlations are about -.12 . The alcohol variables most useful for this predictive purpose are BINGE, ADOCC, MAX, and QFV - measures of binge drinking, with somewhat greater weight on those reflecting the period prior to awareness of pregnancy. Binge-related scores for the period during pregnancy generally enter with decreasing weights.

Corresponding to this regularity in the meaning of the alcohol exposure scale is a surprising regularity in the meaning of the latent variables "targeted" by that scale. In the same way that we scaled alcohol and each outcome against each other in separate two-block analyses, Tables $3 \mathrm{a}$ and $3 \mathrm{~b}$, we also considered the structure of mutual predictability between the outcome blocks in pairs. In all cases, a single pair of latent variables was sufficient to summarize at least $98 \%$ of the relation between the blocks, and these latent variables had all coefficients (except for the mean reaction time item in the Vigilance block) positive and approximately equal. The correlations among all these nearly unweighted averages, drawn over appropriate connecting lines in Fig. 3, range from .35 (Achievement with Vigilance) to .67 (Achievement with IQ).

We had not expected the simplicity of this finding. The relation of alcohol to each of the four outcome blocks is "the same"roughly the same alcohol subscale is the appropriate predictor for explaining covariances with each of the four outcome blocks taken separately. Furthermore, each outcome block has a very simple 


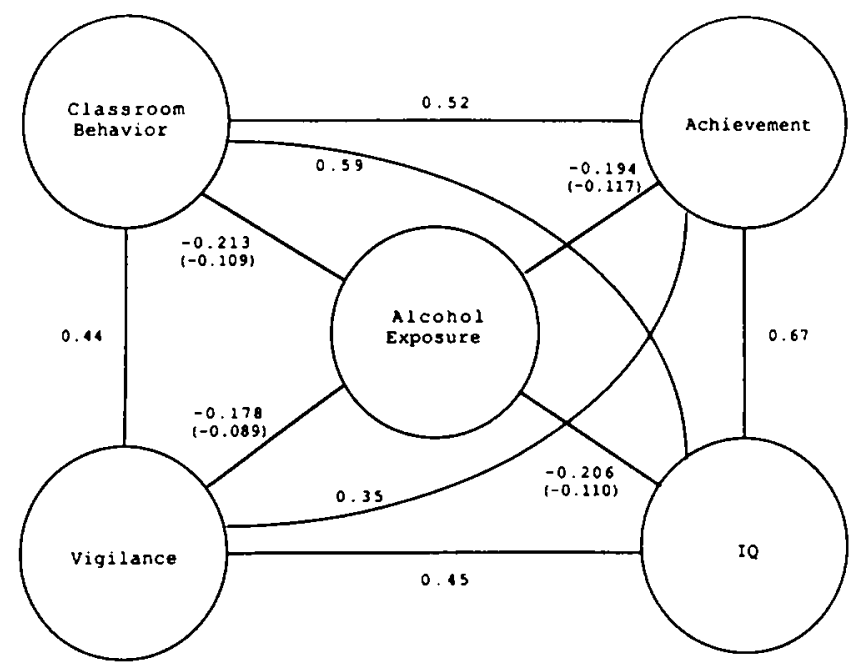

FIG. 3. Diagram demonstrating all two-block latent variable correlations involving Classroom Behavior, Achievement, IQ. Vigilance, and Alcohol Exposure. Numbers in parentheses are the latent variable correlations after adjustment for the covariates described in the text and listed in Table 3b.

structure of predictability by every other outcome block: a single latent variable in each block suffices to explain most of the covariances between any pair of outcome indicators. We may, therefore, ask a final question summarizing the interrelationships of all five blocks: is there a single variable summarizing the effects of alcohol upon the items of all four blocks of indicators? Applying the PLS procedure as usual to the complete list of all 43 outcome indices, we find that the usual alcohol latent variableweighted most heavily on the binge-related measures of prepregnancy recognition consumption - is correlated -.241 with a linear combination of all 43 outcomes having large weights for just those items heavily weighted in the two-block analyses: $\log (\mathrm{AXEC})$, Cooperation/Impulsivity, Digit Span, Arithmetic Achievement, and so forth as shown in Table 5 . This single pair of scales explains $92 \%$ of all the predictability relating alcohol to all 43 of the outcomes, and each block is represented in this single weighted sum by the same items which are emphasized in the study of their relationships to alcohol separately.

The coefficient -.24 is thus a fair summary of the predictive implications of alcohol exposure for the entire assortment of behavioral outcomes measured at age 7 years. We take this value as our best estimate of the "underlying correlation" between learning difficulties, variously measured, and net alcohol exposure as measured indirectly by the alcohol LV. Adjustment for father's education reduces this correlation to -.17 . Adjustment for prenatal and postnatal covariates drops this correlation first to -.14 , then -.12 , consistent with the results of the smaller two-block analyses.

The scatter diagrams corresponding to all the block predictions are similar to each other and to Fig. 4. Keeping in mind the nature of the nonlinear alcohol transformations shown in Fig. 1, we see that low levels of alcohol exposure as measured by all of the 13 original alcohol scores appear to have little import for the outcome latent variables. The effect of the higher alcohol exposures on these outcomes is modest but quite systematic over all four outcome blocks.

\section{DISCUSSION}

The PLS analyses demonstrate that the most salient aspect of

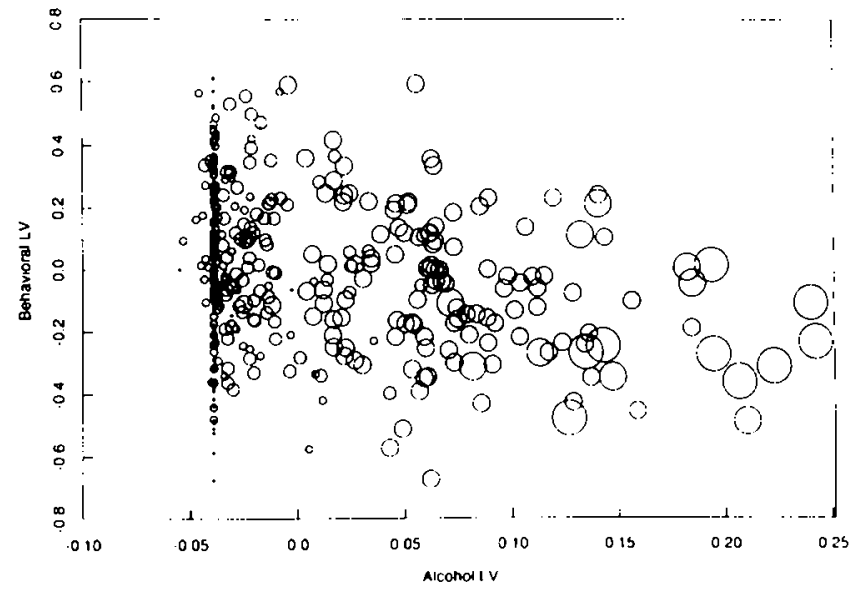

FIG. 4. Scatterplot of scores of the estimated Alcohol LV versus the scores of the estimated Behavioral LV for the two-block model relating 13 indicators of alcohol exposure to all 43 behavioral indicators pooled from the four blocks represented in Fig. 3. The circles plotted have radii proportional to $\log (\mathrm{MAXP})$. The vertical streak at the left of this scatter incorporates all the women who reported no alcohol consumption on any of the alcohol scores. The scattering of points to their left are due to the slight transformations of AAD and MOCCD opposite to those for all other alcohol scores.

prenatal alcohol exposure in terms of predicting behavior and performance in early school-age children is a binge pattern of exposure, particularly in the earliest prenatal period. Three binge scores were the best predictors: ever reporting five or more drinks on any occasion (BINGE), maximum drinks per drinking occasion (MAX), and average number of drinks per drinking occasion (ADOCC). The frequency of drinking occasions is not an important predictor compared to the binge patterns, and the period prior to pregnancy recognition is generally more salient for predicting performance and behavior in the early school-age child than is drinking in the midpregnancy period. (This study did not address the question of late pregnancy drinking.)

These deleterious drinking patterns should be viewed in light of certain sample characteristics. These mothers were generally at low risk for poor pregnancy outcome: primarily middle-class, all were receiving prenatal care by the 5 th month of pregnancy. Only six out of 486 reported any serious alcohol-related problems. On the other hand, the primary binge-drinking score (ever reporting five or more drinks per occasion in the month prior to pregnancy recognition) identified $29 \%$ of the mothers of the 486 children in the follow-up cohort, and $15 \%$ of the original screening sample of 1529 pregnant women from which the follow-up cohort was selected in 1974-75.

These PLS analyses have permitted evaluation of domainspecific behaviors that would have been difficult to identify and assess using conventional techniques of multiple regression. Over the 43 scores comprising the four main outcome tests (IQ and Achievement tests, the Pupil Rating Scale, and the Continuous Performance Test of attention/vigilance), the behaviors predicted by prenatal alcohol share considerable face validity: short-term memory, impulsivity, problems with quantitative functioning, and sustained attention. Manifestations of short-term memory problems are observed in the Digit Span and Information subtests of the WISC-R and in the Retains Information item of the classroom behavior ratings. The impulsivity domain is represented by the predominance of errors on the AX subtest of the CPT vigilance paradigm and by Cooperation/Impulsivity on the PRS. Children 
TABLE 5

SUMMARY OF THE COMPRERTANSIVE 2-BLOCK PLS ANALYSIS

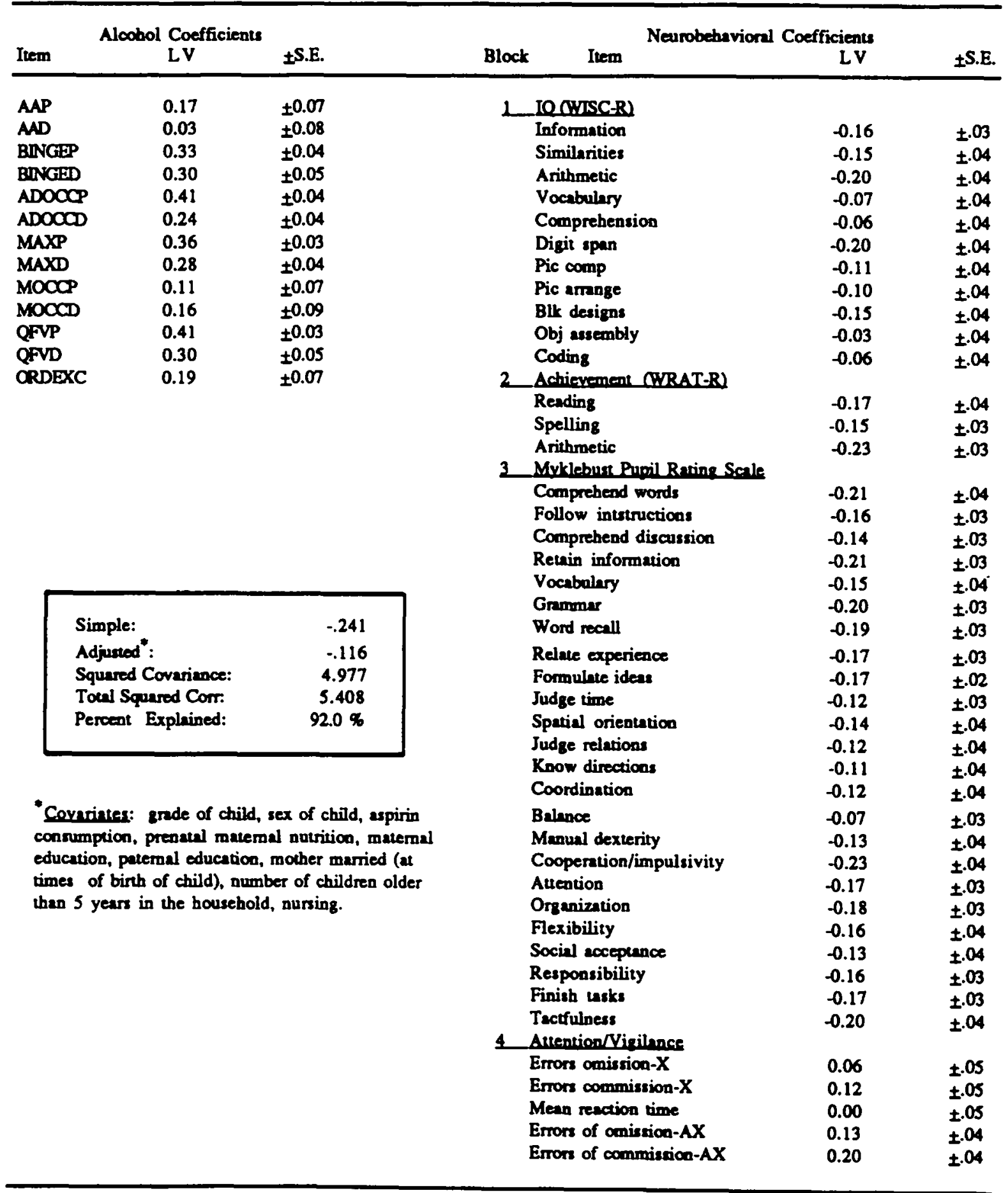

Nowe: $\quad S E=$ Bookstrap Sundard Error

See text Part I [24] for a fuller description of these scores and perinent references.

Alcohol Scores: AA is a continuous variable; $A A>1.00=$ average of $>2$ drinks per day of wine, beer, liquor, or combination.

BINGE is a dichocomous variable represeating whether or not 5 or more drinks were reported on at least one occasion. ADOCC represents the average number of drinks reported per drinking occasion.

MAX is the maximum number of drinks reported for any drinking occarion.

MOCC is the number of occasions per month in which drinking is reported.

QFV is a three dimensional categorical score (Qunatity, Frequency, Variability) deriving from Cahalan, but the order has been reversed for consistency with the other drinking scales, so that 5 is the heaviest. 
TABLE 5

(CONTINUED)

\begin{abstract}
ORDEXC is an a priori code (Ordered Experimental Categories) developed at the outset of this study to describe the presumed risk to the feus of different drinking pattems, in order to enroll women in the follow up study. A score of 4 represents the highest presumed risk.

$P$ refers to the month or so prior to pregnancy recognition, D to drinking during mid-pregnancy, assessed at the Sth month of pregnancy.
\end{abstract}

whose mothers were binge drinkers had more errors on tasks that required withholding a response to certain stimuli (AXEC, and to a lesser extent XEC). Difficulties with quantitative functioning were observed correlated with alcohol exposure on the quantitative subtests of both the intelligence test and the achievement test. Problems with attention for these children may be inferred from the Digit Span subtest of the intelligence test and from poorer performance on the CPT vigilance test.

These relationships between prenatal alcohol exposure and behavioral outcomes are neither explained away nor substantially modified by adjustment for exposure to other drugs such as nicotine, caffeine, aspirin, marijuana and other street drugs. From this list only aspirin consumption was a clearly significant predictor for any of the outcome blocks (primarily the Pupil Rating Scale). Caffeine, nicotine and marijuana consumption are all significantly positively correlated with the alcohol measures, but they are not significantly associated with the outcome LV's. There was some suggestion of possible marijuana effects on the Vigilance and Pupil Rating Scale blocks, but these effects were not clearly significant after adjustment for alcohol exposure and other covariates. Other matemal characteristics, such as nutrition during pregnancy, age, parity or education, race, and other family history variables, failed to affect the relationship between the alcohol and outcome latent variables. However, the most important outcome predictor in our data base, parental education (most saliently measured as paternal education), did consistently explain a large fraction of the unadjusted correlation between the alcohol exposure and performance deficit latent variables.

We have demonstrated here the usefulness of PLS procedures for summarizing relationships among structured blocks of variables such as are commonly measured in observational developmental studies. These procedures are particularly valuable for studies resulting in multiple predictor variables, multiple outcome variables, or both. Specific applications should include behavioral teratology studies, which often have several related estimates of exposure, and long-term outcome studies evaluating the effects of early events (such as low birth weight, prematurity or intracranial hemorrhage) on later outcomes assessed at different ages and across different modalities (such as measures of growth, mental development, motor development, hyperactivity, language delays, etc.). PLS estimation of latent variables for such large, complex data sets averages out the measurement error in correlated blocks of variables while maintaining the "signal," the correlation between latent variables. It handles indefinitely many variables, and it does so without the restrictive assumptions, such as joint normality for the blocks of variables, that prove so unrealistic in typically complex developmental studies.

\title{
REFERENCES
}

1. Becker, R. A.; Chambers, J. M. S: An interactive environment for data analysis and graphics. Belmont, CA: Wadsworth, Inc.: 1984.

2. Bookstein, F. L. The geometric meaning of soft modeling, with some generalizations. In: Joreskog, K. G.; Wold, H., eds. Systems under indirect observation: Causality-structure-prediction, vol. II. Amsterdam: North-Holland Publishing Company; 1982:55-74.

3. Bookstein, F. L. The elements of latent variable models. Adv. Dev. Psychol. 4:203-230; 1986.

4. Bookstein, F. L.; Chernoff, B.; Elder, R.; Humphries, J.; Smith, G.; Strauss, R. Morphometrics in evolutionary biology. (Special Publication 15). Philadelphia: The Academy of Natural Sciences; 1985.

5. Breiman, L.; Friedman, J. H. Estimating optimal transformations for multiple regression and correlation (with discussion). J. Am. Stat. Assoc. 80:580-619:1985.

6. Cochran, W. G. In: Moses, L. E.; Mosteller, F., eds. Planning and analysis of observational studies. New York: John Wiley \& Sons; 1983.

7. Dempster, A. P.; Laird, N. M.; Rubin, D. B. Maximum likelihood from incomplete data via the EM algorithm (with discussion). J. R. Stat. Soc. (Series B) 39:1-38: 1977.

8. Edgington, E. S. Randomization tests. New York: Marcel Dekker; 1980.

9. Efron, B.; Tibshirani, R. Bootstrap methods for standard errors, confidence intervals, and other measures of statistical accuracy. Stat. Sci. 1:54-77; 1986.

10. Fornell. C., ed. A second generation of multivariate analysis, vol. I. New York: Praeger: 1982.

11. Hastie, R.; Tibshirani. T. Generalized additive models (with comments). Stat. Sci. 1(3):297-318; 1986.

12. Hinkley, D. V. Improving the jackknife with special reference to correlation estimation. Biometrika 65:13-21; 1978.
13. Jastak, J. F.; Jastak, S. The wide range achievement test, revised Wilmington, DE: Jastak Associates, Inc.; 1978.

14. Joreskog, K. G.: Sorbon, D. LISREL VI: Analysis of linear structural relationships by maximum likelihood, instrumental variables, and least squares method, 3rd ed. Mooresville, IN: Scientific Software; 1984.

15. Joreskog, K. G.; Wold, H., eds. Systems under indirect observation: Causality-structure-prediction, part I \& part Il. Amsterdam: North Holland Publishing Co.; 1982.

16. Lohmöller, J-B. LVPLS program manual: Latent variables path analysis with partial least-square estimation. Köln: Zentralarchiv für Empirische Sozialforschung. Universität zu Köln; 1984. (Available in the USA from Dr. J. J. McArdle, 1929 Lewis Mountain Rd., Charlottesville, VA 22901.)

17. Lunneborg, C. E. Estimating the correlation coefficient: The bootstrap approach. Psychol. Bull. 98:209-215; 1985.

18. Mardia, K. V.; Kent, J. T.; Bibby, J. M. Multivariate analysis. New York: Academic Press; 1979.

19. Myklebust, H. R. The pupil rating scale revised: Screening for learning disabilities. New York: Grune \& Stratton; 1981.

20. Rosvold, H. E.; Mirsky, A. F.; Sarason, I.; Bransome, E. D., Jr.; Beck, L. H. A continuous performance test of brain damage. J. Consult. Clin. Psychol. 20(5):343-350; 1956.

21. Sampson, P. D.: Streissguth, A. P.; Vega-Gonzalez, S. C.: Barr, H. M.; Bookstein, F. L. Predicting classroom behavior ratings by prenatal alcohol exposure: Latent variable modeling and non-linear scaling. Seattle: University of Washington, Dept. of Statistics, Technical Report \# 103; 1987.

22. Streissguth, A. P. Smoking and drinking during pregnancy and offspring learning disabilities: A review of the literature and development of research strategy. In: Lewis, M., ed. Learning disabilities and 
prenatal risk. Urbana-Champaign, IL: University of Illinois Press; 1986:28-67.

23. Streissguth, A. P.; Barr, H. M.; Martin, D. C.; Darby, B. L. The fetal alcohol syndrome as a model for the study of the behavioral teratology of alcohol. In: Krasnegor, N. A.; Gray, D. B.; Thompson, T., eds. Developmental behavioral pharmacology, vol. 5 . Hillsdale, NJ: Lawrence Erlbaum Assoc., 1986:265-291.

24. Streissguth, A. P.; Barr, H. M.; Sampson, P. D.; Bookstein, F. L.; Darby, B. L. Neurobehavioral effects of prenatal alcohol: Part I. Research strategy. Neurotoxicol. Teratol. 11:461-476; 1989.

25. Streissguth, A. P.; Barr, H. M.; Sampson, P. D.; Parrish-Johnson, J. C.; Kirchner, G. L.; Martin, D. C. Attention, distraction and reaction time at age 7 years and prenatal alcohol exposure. Neurobehav. Toxicol. Teratol. 8(6):717-725; 1986.

26. Streissguth, A. P.; Bookstein, F. L.; Sampson, P. D.; Barr, H. M. Neurobehavioral effects of prenatal alcohol: Part III. PLS analyses of neuropsychologic tests. Neurotoxicol. Teratol. 11:493-507; 1989.

27. Streissguth, A. P.; Martin, D. C.; Buffington, V. E. Test-retest reliability of three scales deriving from a quantity-frequency-variability assessment of self-reported alcohol consumption. Ann. NY Acad. Sci. 273:458-466; 1976.

28. Streissguth, A. P.; Martin, D. C.; Buffington, V. E. Identifying heavy drinkers: A comparison of light alcohol scores obtained on the same sample. In: Seixas, F. A., ed. Current in alcoholism, vol. 2. New York: Grune \& Stramon, Inc.: 1977.

29. Streissguth, A. P.; Martin, D. C.; Martin, J. C.: Barr, H. M. The Seattle longitudinal prospective study on alcohol and pregnancy. Neurobehav. Toxicol. Teratol. 3:223-233; 1981.

30. Streissguth, A. P.; Sampson, P. D.; Barr, H. M.; Clarren, S. K.; Martin, D. C. Studying alcohol teratogenesis from the perspective of the fetal alcohol syndrome: Methodological and statistical issues. In: Snider, D. A., ed. Mental retardation and developmental disabilities Research, education and technology transfer. New York: New York Academy of Sciences; 1986.

31. Torgerson, W. S. Theory and methods of scaling. New York: Wiley \& Sons; 1958.

32. Wechsler, D. The Wechsler intelligence scale for children, revised. New York: The Psychological Corporation; 1974.

33. Wold, H. Path models with latent variables: The NIPALS approach In: Blalock, H. M., et al., eds. Quantitative sociology: International perspectives on mathematical and statistical modeling. New York: Academic Press; 1975:307-357.

34. Wold, H. Soft modeling: The basic design and some extensions. In Systems under indirect observation: Causality-structure-prediction, vol. 2. Amsterdam: North Holland Publishing Company; 1982:1-54.

35. Wold, H., ed. Theoretical empiricism. New York: Paragon House; 1989.

\section{APPENDIX}

An alternative numerical approach to solving the equations (1) and (2) follows from reexpressing the equations in matrix notation. Let $\alpha$ denote the column vector comprised of $\left(\alpha_{1}, \ldots, \alpha_{13}\right)$, and $\beta$ denote the column vector of $\left(\beta_{1}, \ldots, \beta_{11}\right)$. Then, equation (1) becomes

$$
\alpha \propto R_{\mathrm{AB}} \beta \text {, }
$$

(where " $x$ " means "is proportional to"), and (2) becomes

$$
\beta \propto \mathbf{R}_{\mathrm{BA}} \alpha
$$

Substituting (A.2) into (A.1) we obtain

$$
\alpha \propto \mathbf{R}_{\mathrm{AB}} \mathbf{R}_{\mathrm{BA}} \alpha
$$

and substituting (A.1) into (A.2),

$$
\beta \propto R_{B A} R_{A B} \beta .
$$

These equations define $\alpha$ and $\beta$ as eigenvectors of $R_{A B} R_{B A}$ and $R_{B A} R_{A B}$, respectively. They may be computed more conveniently as left and right "singular vectors" of $R_{A B}(31)$ :

$$
\mathbf{R}_{\mathrm{AB}}=\mathrm{UDV}^{\mathrm{T}}
$$

where $U$ is a $13 \times 11$ matrix of (orthonormal) left singular vectors, $\mathrm{V}$ is an $11 \times 11$ matrix of right singular vectors, and $\mathrm{D}=$ $\operatorname{diag}\left(d_{1}, \ldots, d_{11}\right)$ is an $11 \times 11$ diagonal matrix of "singular values." The singular values provide the covariance between the linear combinations $A^{T} u_{i}$ and $B^{T} v_{i}$ where $A$ and $B$ are the vectors of alcohol and IQ subtest scores, and $u_{i}$ and $v_{i}$, denote columnns of $U$ and $V$, respectively. The coefficient vectors we seek are then $\alpha=u_{1}$ and $\beta=v_{1}$, the linear combinations with maximum covariance. Further linear combinations or latent variables, computed from subsequent singular vectors, may be considered if the first linear combinations do not adequately explain the correlation matrix $R_{A B}$ (or, equivalently, if the first singular value $d_{1}$ does not greatly dominate the entire vector of singular values).

To complete our contrast of this PLS analysis with canonical correlation we note that the characteristic equations yielding canonical variable coefficients are

$$
\alpha \propto\left(\mathrm{R}_{\mathrm{AA}}\right)^{-1} \mathrm{R}_{\mathrm{AB}}\left(\mathrm{R}_{\mathrm{BB}}\right)^{-1} \mathrm{R}_{\mathrm{BA}} \alpha
$$

and

$$
\beta \propto\left(R_{B B}\right)^{-1} R_{B A}\left(R_{A A}\right)^{-1} R_{A B} \beta .
$$

Note the dependence of the result on the within-block correlation matrices $R_{A A}$ and $R_{B B}$. When there is a high degree of collinearity within blocks, the inverses of these matrices (and the corresponding canonical variable coefficients) are unstable.

The computations described here can be easily programmed with the aid of numerical analysis subroutines for computing eigenvectors or singular value decompositions. (These are available in a number of widely available mathematical subroutine libraries, such as EISPACK, IMSL, and NAG.) A commerical program (16) can also be used for these simple two-block analyses (and for much more complicated PLS models). Researchers with access to a computer running the UNIX operating system and the "S" package for data analysis and graphics (1) may contact the lead author (P.D.S.) for special purpose programs for the analyses described here. 